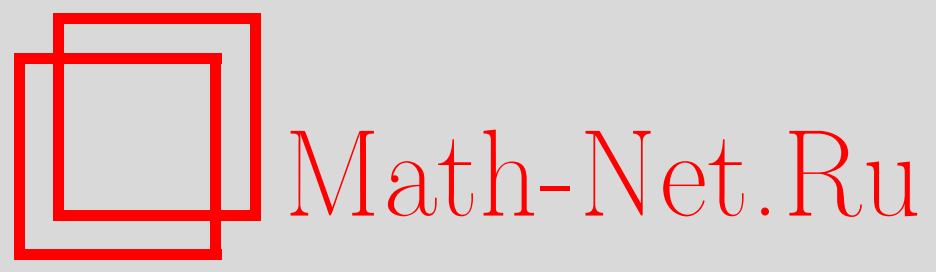

Д. В. Ховратович, О мощности некоторых подклассов монотонных функций, Дискрет. матем., 2005, том 17, выпуск 4, 81-97

DOI: https://doi.org/10.4213/dm131

Использование Общероссийского математического портала Math-Net.Ru подразумевает, что вы прочитали и согласны с пользовательским соглашением http: //www . mathnet.ru/rus/agreement

Параметры загрузки:

IP: 3.82 .47 .9

26 апреля 2023 г., 15:38:58 


\title{
О мощности некоторых подклассов монотонных функций
}

\author{
() 2005 г. Д. В. Ховратович
}

\begin{abstract}
Рассматривается вопрос о числе функций от $n$ переменных из классов, являющихся пересечениями класса $M$ монотонных функций с другими предполными классами в трехзначной логике. Получена асимптотика при $n \rightarrow \infty$ логарифма мощности таких классов.
\end{abstract}

\section{1. Общий подход}

Задача оценки числа функщий из некоторого класса $\mathscr{F}$ может быть решена следующим способом.

Каждой функции $f \in \mathscr{F}$ по некоторому правилу ставится в соответствие набор функций $\left\langle f_{1}, f_{2}, \ldots, f_{l}\right\rangle$. Это соответствие можно описать как отображение

$$
\mathscr{P}: \mathscr{F} \rightarrow \widehat{\mathscr{F}}_{1} \times \widehat{\mathscr{F}}_{2} \times \ldots \times \widehat{\mathscr{F}}_{l},
$$

где $\widehat{\mathscr{F}}_{i}, i=1, \ldots, n,-$ некоторые множества функций, символ $\times$ обозначает взятие декартова произведения множеств. Тогда, если отображение $\mathscr{P}$ инъективно, то

$$
|\mathscr{F}| \leqslant\left|\widehat{\mathscr{F}}_{1}\right|\left|\widehat{\mathscr{F}}_{2}\right| \ldots\left|\widehat{\mathscr{F}}_{l}\right| \text {. }
$$

Если известны верхние оценки мощности классов $\widehat{\mathscr{F}}_{i}$, то можно легко получить верхнюю оценку мощности $\mathscr{F}$.

С учетом уже построенных множеств $\widehat{\mathscr{F}}_{i}$, для получения нижней оценки достаточно, чтобы для некоторого $k, 1 \leqslant k \leqslant l$, существовало инъективное отображение 2, действующее из $\widehat{\mathscr{F}}_{k}$ в $\mathscr{F}$. Тогда $|\mathscr{F}| \geqslant\left|\widehat{\mathscr{F}}_{k}\right|$.

\section{2. Основные определения}

Обозначим через $E_{k}, k \in \mathbf{N}$, множество $\{0,1, \ldots, k-1\}, n$-ю декартову степень этого множества обозначим через $E_{k}^{n}$, а элементы полученного множества будем называть наборами гиперкуба (или просто куба) и представлять в виде $\left(a_{1}, \ldots, a_{n}\right)$.

Далее для сокращения записи будем обозначать через $f(A)$ множество всех значений, принимаемых функщией $f$ на множестве $A$. Множество функщий, действующих из $A$ в $B$, будем обозначать $\{A \rightarrow B\}$. 
Определение 1. Пусть $j \in E_{k}$. Тогда назовем $j$-подкубом куба $E_{k}^{n}$ с индексом $N \subset\{1,2, \ldots, n\}$ множество всех таких наборов $\left(\alpha_{1}, \ldots, \alpha_{n}\right)$ куба $E_{k}^{n}$, что

$$
\alpha_{i}=j \Longleftrightarrow i \in N, \quad i=1, \ldots n
$$

Например, 2-подкуб куба $E_{3}^{3}$ с индексом $\{2,3\}$ - это множество наборов $\{(0,2,2)$, $(1,2,2)\}$.

Замечание 1. Заметим, что число $j$-подкубов в кубе $E_{k}^{n}$ не зависит от $k$ и равно $2^{n}$. Кроме того, $j$-подкубы не пересекаются, а в объединении дают весь куб $E_{k}^{n}$.

Определение 2. Частичным порядком $O$ на множестве $E_{k}$ называется двухместное отношение на этом множестве, удовлетворяющее условиям рефлексивности, транзитивности и антисимметричности. Для элементов из $E_{k}$, удовлетворяющих этому отношению, применяется обозначение $a \leqslant o b$, причем, если $a \neq b$, то будем писать $a<b$. Если любые два элемента сравнимы по данному порядку, то он называется линейным. В дальнейшем будем рассматривать только линейные порядки.

Введем обозначения для линейных порядков, используемых нами в дальнейшем, пусть

$$
\begin{array}{ll}
O_{3}: & 0<1<2, \\
O_{3}^{0}: & 0<1, \\
O_{3}^{2}: & 1<2, \\
O_{3}^{\prime}: & 1<0<2 .
\end{array}
$$

Определение 3. Пусть на множестве $E_{k}$ задан порядок $O$, а наборы $a$ и $b$ принадлежат множеству $E_{k}^{n}$. Будем говорить, что набор $a=\left(a_{1}, a_{2}, \ldots, a_{n}\right)$ меньше либо равен набору $b=\left(b_{1}, b_{2}, \ldots, b_{n}\right)$ согласно порядку $O$ и писать $a \leqslant o b$, если $a_{i} \leqslant o b_{i}, i=1, \ldots n$. Легко проверить, что заданное таким образом отношение также является порядком.

Определение 4. Пусть на множестве $E_{k}$ задан некоторый порядок $O$. Функщии $f$, действующие из $E_{k}^{n}$ в $E_{k}$ и такие, что

$$
\forall a, b \in E_{k}^{n} \quad a \leqslant o b \Longrightarrow f(a) \leqslant o f(b)
$$

будем называть сохраняющими порядок $O$, а класс всех таких функщий обозначать $\{A \rightarrow O B\}$.

Определение 5. Классом $M_{3}(n)$ называется множество функций $\left\{E_{3}^{n} \rightarrow O_{3} E_{3}\right\}$. Известно [1], что данный класс является предполным в множестве $\mathscr{P}_{3}$ всех функщй трехзначной логики.

Определение 6. Пусть на множестве $A$ введен некоторый порядок $O_{1}$, а на множестве $B$ - некоторый порядок $O_{2}$. Функщии $f$, действующие из $A$ в $B$ и такие, что

$$
\forall a, b \in A \quad a \leqslant o_{1} b \Longrightarrow f(a) \leqslant o_{2} f(b)
$$

будем называть сохраняющими порядок $O_{1} \mid O_{2}$, а класс всех таких функций обозначать $\left\{A \rightarrow O_{1} \mid O_{2} B\right\}$. 


\section{3. Класс $M_{3}(n) \cap U_{\{0\}\{1,2\}}(n)$}

Определение 7. Классом $U_{\{0\}\{1,2\}}(n)$ называется множество всех функщий трехзначной логики от $n$ переменных, сохраняюших разбиение $D=\{0\}\{1,2\}$ (см. также [1]).

Согласно [1], данный класс является предполным в $\mathscr{P}_{3}$.

Теорема 1. Для числа $\psi_{M U_{0}}(n)$ функций в классе $M_{3}(n) \cap U_{\{0\}\{1,2\}}(n)$ справедливо равенство

$$
\psi_{M U_{0}}(n)=2^{\sqrt{3 /(4 \pi n)} 3^{n}(1+\varepsilon(n))},
$$

где $\varepsilon(n) \rightarrow 0$ npu $n \rightarrow \infty$.

Для доказательства теоремы нам потребуется несколько вспомогательных утверждений.

Обозначим исследуемый класс функщий через $\mathscr{F}$.

Лемма 1. Пусть $f \in \mathscr{F}$. Тогда на каждом 0-подкубе куба $E_{3}^{n}$ функция $f$ принимает либо только значение 0, либо только значения 1 и 2 .

Доказательство. Пусть $A$ - некоторый 0-подкуб куба $E_{3}^{n}$, а $N$ - его индекс. Заметим, что любые два набора $\widetilde{\alpha}$ и $\widetilde{\beta}$ из $A$ эквивалентны согласно данному разбиению, это следует из того, что

$$
\tilde{\alpha}_{i}=0 \Longleftrightarrow i \in N \Longleftrightarrow \tilde{\beta}_{i}=0 \text {. }
$$

Функция $f$ сохраняет разбиение $D$, поэтому

$$
\forall \tilde{\alpha}, \tilde{\beta} \in A \quad f(\tilde{\alpha}) \stackrel{D}{\sim} f(\tilde{\beta}),
$$

следовательно, на наборах из $A$ функция $f$ принимает либо только значение 0 , либо только 1 и 2. Отсюда следует утверждение леммы.

Введем множество $\hat{A}=\left\{A_{1}, A_{2}, \ldots, A_{2^{n}}\right\}$, состоящее из всех 0-подкубов куба $E_{3}^{n}$. Заметим, что $\bigcup_{A_{i} \in A} A_{i}=E_{3}^{n}$.

Введем также множество $\widehat{S}=\left\{S_{1}, S_{2}\right\}$ подстановок на $E_{k}$, где

$$
S_{1}=\left(\begin{array}{lll}
0 & 1 & 2 \\
1 & 1 & 2
\end{array}\right), \quad S_{2}=\left(\begin{array}{lll}
0 & 1 & 2 \\
0 & 1 & 2
\end{array}\right)
$$

и построим функциональные множества

$$
\begin{aligned}
& \widehat{\mathscr{F}}_{1}=\{\hat{A} \rightarrow \widehat{S}\}, \\
& \widehat{\mathscr{F}}_{2}=\left\{E_{3}^{n} \rightarrow{ }_{O_{3} \mid O_{3}^{2}}\{1,2\}\right\} .
\end{aligned}
$$

Фиксируем произвольную функщию $f$ из $\mathscr{F}$. Определим для нее функщию $f_{1}$ из $\widehat{\mathscr{F}}_{1}$, полагая для всех $A \in \hat{A}$

$$
f_{1}(A)= \begin{cases}S_{1}, & \text { если } f(A)=\{0\} \\ S_{2} & \text { в противном случае }\end{cases}
$$

Теперь построим функцию $f_{2}$, определенную на $E_{3}^{n}$, полагая для всех $\tilde{\alpha} \in E_{3}^{n}$

$$
f_{2}(\tilde{\alpha})=f_{1}\left(A_{\tilde{\alpha}}\right)[f(\tilde{\alpha})],
$$

где $A_{\tilde{\alpha}}-0$-подкуб, содержащий набор $\tilde{\alpha}$. Корректность такого определения следует из того, что каждый набор попадает ровно в один 0 -подкуб. 
Лемма 2. Пусть $f$-функция из $\mathscr{F}$, а функции $f_{1}$ и $f_{2}$ задаются по правилам (3) $и$ (4) соответственно. Тогда функиия $f_{2}$ принадлежст функииональному множеству $\widehat{\mathscr{F}}_{2}$.

Доказательство. Согласно определению подстановок из $\widehat{S}$, если $f(\tilde{\alpha}) \neq 0$, то $f(\widetilde{\alpha})=f_{2}(\widetilde{\alpha})$, в противном случае $f_{2}(\widetilde{\alpha})=1$.

Таким образом, функция $f_{2}$ получается из $f$ заменой всех нулей на единицы. Из этого следует, что $f_{2}\left(E_{3}^{n}\right) \subset\{1,2\}$. Теперь возьмем два произвольных набора $a$ и $b$ из $E_{3}^{n}$ такие, что $a \leqslant O_{3} b$. Функция $f$ сохраняет порядок $O_{3}$, поэтому

$$
f(a) \leqslant o_{3} f(b) \Longrightarrow f_{2}(a) \leqslant o_{3} f_{2}(b) .
$$

Из этого следует, что $f_{2}(a) \leqslant o_{3}^{2} f_{2}(b)$. Лемма доказана.

Лемма 3. Существует инъективное отображение $\mathscr{P}$ множества $\mathscr{F}_{в} \widehat{\mathscr{F}}_{1} \times \widehat{\mathscr{F}}_{2}$.

Доказательство. Положим $\mathscr{P}(f)=\left(f_{1}, f_{2}\right)$, где функщии $f_{1}$ и $f_{2}$ задаются по правилам (3) и (4) соответственно. Функщия $f_{1}$ принадлежит множеству $\widehat{\mathscr{F}}_{1}$ по определению. Функция $f_{2}$ принадлежит множеству $\widehat{\mathscr{F}}_{2}$ по лемме 2. Таким образом, отображение $\mathscr{P}$ действует из $\mathscr{F}_{\text {в }} \widehat{\mathscr{F}}_{1} \times \widehat{\mathscr{F}}_{2}$.

Покажем его инъективность. Действительно, пусть функии $f^{\prime}$ и $f^{\prime \prime}$ принадлежат классу $\mathscr{F}$ и отличны друг от друга на наборе $\widetilde{\alpha}$. Возможны следующие варианты.

Среди значений $f^{\prime}(\widetilde{\alpha}), f^{\prime \prime}(\widetilde{\alpha})$ есть 2 . Тогда $f_{2}^{\prime}(\widetilde{\alpha}) \neq f_{2}^{\prime \prime}(\widetilde{\alpha})$, так как подстановки множества $\widehat{S}$ переводят в двойку только двойку, поэтому $\mathscr{P}\left(f^{\prime}\right) \neq \mathscr{P}\left(f^{\prime \prime}\right)$.

Среди значений $f^{\prime}(\widetilde{\alpha}), f^{\prime \prime}(\tilde{\alpha})$ нет двойки, а значит, есть 0 и 1 . Пусть $A_{\tilde{\alpha}}$ есть 0 -подкуб, соответствующий набору $\tilde{\alpha}$. Тогда, по лемме 1 , на этом подкубе одна из функций будет принимать только значение 0 , а другая - только 1 и 2 . Отсюда следует, что $f_{1}^{\prime}\left(A_{\tilde{\alpha}}\right) \neq f_{1}^{\prime \prime}\left(A_{\tilde{\alpha}}\right)$. Таким образом, и в этом случае $\mathscr{P}\left(f^{\prime}\right) \neq \mathscr{P}\left(f^{\prime \prime}\right)$. Лемма доказана.

Лемма 4. Справедлива оченка

$$
|F| \leqslant 2^{\sqrt{3 /(4 \pi n)} 3^{n}\left(1+\varepsilon_{1}(n)\right)},
$$

где $\varepsilon_{1}(n) \rightarrow 0$ npu $n \rightarrow \infty$.

Доказательство. Согласно лемме 3 существует инъективное отображение $\mathscr{P}$, действую-

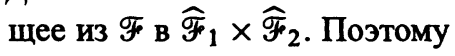

$$
|\mathscr{F}|=|\mathscr{P}(\mathscr{F})| \leqslant\left|\widehat{\mathscr{F}}_{1}\right|\left|\widehat{\mathscr{F}}_{2}\right| \text {. }
$$

Мощность $\widehat{\mathscr{F}}_{1}$, очевидно, равна $|\hat{A}|^{|\hat{S}|}=2^{2^{n}}$, а для мощности $\widehat{\mathscr{F}}_{2}$ справедлива оценка (cM. [2])

$$
\left|\widehat{\mathscr{F}}_{2}\right|=2^{\sqrt{3 /(4 \pi n)} 3^{n}\left(1+\varepsilon_{2}(n)\right)},
$$

где $\varepsilon_{2}(n) \rightarrow 0$ при $n \rightarrow \infty$.

Отсюда получаем, что

$$
|\mathscr{F}| \leqslant 2^{2^{n}} 2^{\sqrt{3 /(4 \pi n)} 3^{n}\left(1+\varepsilon_{2}(n)\right)}=2^{\sqrt{3 /(4 \pi n)} 3^{n}\left(1+\varepsilon_{2}(n)\right)+2^{n}}=2^{\sqrt{3 /(4 \pi n)} 3^{n}\left(1+\varepsilon_{1}(n)\right)},
$$

где $\varepsilon_{1}(n) \rightarrow 0$ при $n \rightarrow \infty$. Лемма доказана. 
Лемма 5. Существует инъективное отображение 2 из $\widehat{\mathscr{F}}_{2}$ в $\mathscr{F}$.

Доказательство. Покажем, что достаточно взять тривиальное отображение

$$
2\left(f_{2}\right)=f_{2}
$$

для всех $f_{2} \in \widehat{\mathscr{F}}_{2}$. Очевидно, что это отображение инъективно. Покажем, что 2 действует в F. Это утверждение эквивалентно тому, что

$$
\widehat{\mathscr{F}}_{2} \subset M_{3}(n) \cap U_{\{0\}\{1,2\}}(n) .
$$

Действительно, функщии из $\widehat{\mathscr{F}}_{2}$ сохраняют порядок $O_{3}$ : из того, что $f_{2}(a) \leqslant O_{3}^{2} f_{2}(b)$ при всех $a \leqslant o_{3} b$, следует, что $f_{2}(a) \leqslant o_{3} f_{2}(b)$. Они также сохраняют разбиение $\{0\}\{1,2\}$, так как множество значений этих функщий попадает в один класс эквивалентности по данному разбиению.

Лемма 6. Справедлива оченка

$$
|\mathscr{F}| \geqslant 2^{\sqrt{3 / 4 \pi n} 3^{n}\left(1+\varepsilon_{2}(n)\right)},
$$

где $\varepsilon_{2}(n) \rightarrow 0$ npu $n \rightarrow \infty$.

Доказательство. Согласно лемме 5 существует инъективное отображение 2, действующее из $\widehat{\mathscr{F}}_{2}$ в $\mathscr{F}$. Поэтому в силу (5)

$$
|\mathscr{F}| \geqslant\left|\widehat{\mathscr{F}}_{2}\right|=2^{\sqrt{3 /(4 \pi n)} 3^{n}\left(1+\varepsilon_{2}(n)\right)} .
$$

Утверждение теоремы следует теперь из лемм 4 и 6.

Следствие 1. Пусть $\psi_{M U_{1}}(n)-$ число функций из класса $M_{3}(n) \cap U_{\{1\}\{0,2\}}(n), a \psi_{M U_{2}}(n)$ - число функций из класса $M_{3}(n) \cap U_{\{2\}\{0,1\}}(n)$. Тогда справедливы соотночения

$$
\begin{aligned}
& \psi_{M U_{1}}(n)=2^{\sqrt{3 /(4 \pi n) 3^{n}}\left(1+\varepsilon_{3}(n)\right)}, \\
& \psi_{M U_{2}}(n)=2^{\sqrt{3 /(4 \pi n)} 3^{n}\left(1+\varepsilon_{4}(n)\right)},
\end{aligned}
$$

где $\varepsilon_{3}(n) \rightarrow 0 u \varepsilon_{4}(n) \rightarrow 0$ npu $n \rightarrow \infty$.

Для доказательства этих соотношений достаточно провести те же самые рассуждения с перестановкой констант и изменением соответствующих порядков.

\section{4. Класс $M_{3}(n) \cap T_{\mathscr{E}_{0}, 1}(n)$}

Определение 8. Классом $T_{\mathscr{E}_{0}, 1}(n)$ называется множество всех функций трехзначной логики от $\boldsymbol{n}$ переменных, сохраняющих предикат

$$
R_{1}=\left(\begin{array}{lllllll}
0 & 0 & 0 & 1 & 1 & 2 & 2 \\
0 & 1 & 2 & 0 & 1 & 0 & 2
\end{array}\right)
$$

Согласно [1], данный класс является предполным в $\mathscr{P}_{3}$. 
Теорема 2. Для числа $\psi_{M} T_{0}(n)$ функций в классе $M_{3}(n) \cap T_{\mathscr{C}_{0}, 1}(n)$ справедливо равенство

$$
\psi_{M T_{0}}(n)=2^{\sqrt{3 /(4 \pi n)} 3^{n}(1+\varepsilon(n))},
$$

где $\varepsilon(n) \rightarrow 0$ npu $n \rightarrow \infty$.

Для доказательства теоремы нам потребуется несколько вспомогательных утверждений.

Обозначим исследуемый класс функщий через F.

Лемма 7. Пусть $f \in \mathscr{F}, A-$ произвольный 2-подкуб куба $E_{3}^{n}$. Тогда 1 и 2 не входят во множество $f(A)$ одновременно.

Доказательство. Пусть $N$ - индекс 2-подкуба $A$. Заметим, что любые два набора $\tilde{\alpha}$ и $\tilde{\beta}$ из $A$ сравнимы по предикату $R_{1}$, это следует из того, что

$$
\tilde{\alpha}_{i}=2 \Longleftrightarrow i \in N \Longleftrightarrow \tilde{\beta}_{i}=2 \text {, }
$$

поэтому запрещенных по данному предикату пар значений не возникает. Функщия $f$ сохраняет предикат $R_{1}$, поэтому любая пара ее значений на наборах из $A$ должна быть сравнима по данному предикату. Таким образом, невозможен случай, когда на одном наборе принимается значение 1 , а на другом - значение 2 . Из этого следует, что эти два значения не могут входить в множество $f(A)$ одновременно. Отсюда следует утверждение леммы.

Введем множество $\hat{A}=\left\{A_{1}, A_{2}, \ldots, A_{2^{n}}\right\}$, состоящее из всех 2-подкубов куба $E_{3}^{n}$. Заметим, что

$$
\bigcup_{A_{i} \in A} A_{i}=E_{3}^{n}
$$

Введем множество подстановок $\widehat{S}=\left\{S_{1}, S_{2}\right\}$, где

$$
S_{1}=\left(\begin{array}{lll}
0 & 1 & 2 \\
0 & 1 & 1
\end{array}\right), \quad S_{2}=\left(\begin{array}{lll}
0 & 1 & 2 \\
0 & 1 & 2
\end{array}\right),
$$

и построим функщиональные множества

$$
\begin{aligned}
& \widehat{\mathscr{F}}_{1}=\{\hat{A} \rightarrow \widehat{S}\}, \\
& \widehat{\mathscr{F}}_{2}=\left\{E_{3}^{n} \rightarrow{ }_{O_{3} \mid O_{3}^{0}}\{0,1\}\right\} .
\end{aligned}
$$

Фиксируем произвольную функцию $f$ из $\mathscr{F}$. Определим для нее функцию $f_{1}$ из $\widehat{F}_{1}$, полагая для всех $A \in \hat{A}$

$$
f_{1}(A)= \begin{cases}S_{1}, & \text { если } 2 \in f(A), \\ S_{2} & \text { в противном случае. }\end{cases}
$$

Теперь построим функцию $f_{2}$, определенную на $E_{3}^{n}$, полагая для всех $\tilde{\alpha} \in E_{3}^{n}$

$$
f_{2}(\tilde{\alpha})=f_{1}\left(A_{\tilde{\alpha}}\right)[f(\tilde{\alpha})],
$$

где $A_{\tilde{\alpha}}-2$-подкуб, содержащий набор $\tilde{\alpha}$. Корректность такого определения следует из того, что каждый набор попадает ровно в один 2-подкуб. 
Лемма 8. Пусть $f$ - функция из F, а функции $f_{1} u f_{2}$ задаются по правилам (6) $и$ (7) соответственно. Тогда функиия $f_{2}$ принадлежит функциональному множеству $\widehat{\mathscr{F}}_{2}$.

Доказательство. Согласно определению подстановок из $\widehat{S}$, если $f(\widetilde{\alpha}) \neq 2$, то $f(\tilde{\alpha})=f_{2}(\tilde{\alpha})$, в противном случае $f_{2}(\tilde{\alpha})=1$.

Таким образом, функщия $f_{2}$ получается из $f$ заменой всех двоек на единищы. Из этого следует, что $f_{2}\left(E_{3}^{n}\right) \subset\{0,1\}$. Теперь возьмем произвольные два набора $a$ и $b$ из $E_{3}^{n}$ такие, что $a \leqslant o_{3} b$. Функция $f$ сохраняет порядок $O_{3}$, поэтому

$$
f(a) \leqslant o_{3} f(b) \Longrightarrow f_{2}(a) \leqslant o_{3} f_{2}(b) .
$$

Из этого следует, что $f_{2}(a) \leqslant o_{3}^{0} f_{2}(b)$. Лемма доказана.

Лемма 9. Существует инъективное отображение $\mathscr{P}$ из $\mathscr{F}_{в} \widehat{\mathscr{F}}_{1} \times \widehat{\mathscr{F}}_{2}$.

Доказательство. Положим $\mathscr{P}(f)=\left(f_{1}, f_{2}\right)$, где функции $f_{1}$ и $f_{2}$ задаются по правилам (6) и (7) соответственно. Функщия $f_{1}$ принадлежит множеству $\widehat{\mathscr{F}}_{1}$ по определению. Функция $f_{2}$ принадлежит множеству $\widehat{\mathscr{F}}_{2}$ по лемме 8 . Таким образом, отображение $\mathscr{P}$ действует

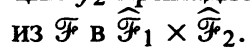

Покажем его инъективность. Действительно, пусть функции $f^{\prime}$ и $f^{\prime \prime}$ принадлежат классу $\mathscr{F}$ и отличны друг от друга на наборе $\widetilde{\alpha}$. Возможны следующие варианты.

Среди значений $f^{\prime}(\widetilde{\alpha}), f^{\prime \prime}(\widetilde{\alpha})$ есть 0 . Тогда $f_{2}^{\prime}(\tilde{\alpha}) \neq f_{2}^{\prime \prime}(\widetilde{\alpha})$, так как подстановки множества $\hat{S}$ переводят в ноль только ноль, поэтому $\mathscr{P}\left(f^{\prime}\right) \neq \mathscr{P}\left(f^{\prime \prime}\right)$.

Среди значений $f^{\prime}(\widetilde{\alpha}), f^{\prime \prime}(\widetilde{\alpha})$ нет нуля, а значит, есть 2 и 1 . Пусть $A_{\tilde{\alpha}}-2$-подкуб, соответствующий набору $\tilde{\alpha}$. Тогда, по лемме 7 , на этом 2-подкубе одна из функций будет принимать значение 2 , а другая не будет. Отсюда следует, что $f_{1}^{\prime}\left(A_{\tilde{\alpha}}\right) \neq f_{1}^{\prime \prime}\left(A_{\tilde{\alpha}}\right)$. Таким образом, и в этом случае $\mathscr{P}\left(f^{\prime}\right) \neq \mathscr{P}\left(f^{\prime \prime}\right)$. Лемма доказана.

Лемма 10. Справедлива оченка

$$
|\mathscr{F}| \leqslant 2^{\sqrt{3 /(4 \pi n)} 3^{n}\left(1+\varepsilon_{1}(n)\right)} .
$$

где $\varepsilon_{1}(n) \rightarrow 0$ npu $n \rightarrow \infty$.

Доказательство. Согласно лемме 9 существует инъективное отображение $\mathscr{P}$, действую-

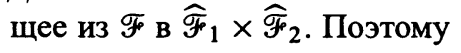

$$
|\mathscr{F}|=|\mathscr{P}(\mathscr{F})| \leqslant\left|\widehat{\mathscr{F}}_{1}\right|\left|\widehat{\mathscr{F}}_{2}\right| .
$$

Мощность $\widehat{\mathscr{F}}_{1}$, очевидно, равна $|\hat{A}|^{|\widehat{S}|}=2^{2^{n}}$, а для мощности $\widehat{\mathscr{F}}_{2}$ справедлива оценка (см. [2])

$$
\left|\widehat{\mathscr{F}}_{2}\right|=2^{\sqrt{3 /(4 \pi n)} 3^{n}\left(1+\varepsilon_{2}(n)\right)},
$$

где $\varepsilon_{2}(n) \rightarrow 0$ при $n \rightarrow \infty$. Отсюда получаем, что

$$
|\mathscr{F}| \leqslant 2^{2^{n}} 2^{\sqrt{3 /(4 \pi n)} 3^{n}\left(1+\varepsilon_{2}(n)\right)}=2^{\sqrt{3 /(4 \pi n)} 3^{n}\left(1+\varepsilon_{2}(n)\right)+2^{n}}=2^{\sqrt{3 /(4 \pi n)} 3^{n}\left(1+\varepsilon_{1}(n)\right)},
$$

где $\varepsilon_{1}(n) \rightarrow 0$ при $n \rightarrow \infty$.

Лемма 11. Существует инъективное отображение 2 из $\widehat{\mathscr{F}}_{2}$ в $\mathscr{F}$. 
Доказательство. Покажем, что достаточно взять тривиальное отображение

$$
2\left(f_{2}\right)=f_{2}
$$

для всех $f_{2} \in \widehat{\mathscr{F}}_{2}$. Очевидно, что это отображение инъективно. Покажем, что 2 действует в F्F. Это утверждение эквивалентно тому, что

$$
\widehat{\mathscr{F}}_{2} \subset M_{3}(n) \cap T_{\mathscr{E}_{0}, 1}(n) .
$$

Действительно, все функции из $\widehat{\mathscr{F}}_{2}$ сохраняют порядок $O_{3}$ : если $f_{2}(a) \leqslant o_{3}^{2} f_{2}(b)$ для всех $a \leqslant o_{3} b$, то $f_{2}(a) \leqslant o_{3} f_{2}(b)$. Они также сохраняют предикат $R_{1}$, так как любая пара значений из множества $\{0,1\}$ удовлетворяет этому предикату.

Лемма 12. Справедлива оченка

$$
|F| \geqslant 2^{\sqrt{3 /(4 \pi n)} 3^{n}\left(1+\varepsilon_{2}(n)\right)},
$$

где $\varepsilon_{2}(n) \rightarrow 0$ npu $n \rightarrow \infty$.

Доказательство. Согласно лемме 11 существует инъективное отображение 2, действующее из $\widehat{F}_{2}$ в $\mathscr{F}$. Поэтому в силу (8)

$$
|\mathscr{F}| \geqslant\left|\widehat{\mathscr{F}}_{2}\right|=2^{\sqrt{3 /(4 \pi n)} 3^{n}\left(1+\varepsilon_{2}(n)\right)} .
$$

Утверждение теоремы следует теперь из лемм 10 и 12.

Следствие 2. Обозначим через $T_{\mathscr{\varepsilon}_{2}, 1}(n)$ класс функций трехзначной логики от $n$ переменных, сохраняющих предикат

$$
R_{1}=\left(\begin{array}{lllllll}
0 & 0 & 0 & 1 & 1 & 2 & 2 \\
0 & 1 & 2 & 0 & 1 & 0 & 2
\end{array}\right)
$$

Пусть $\psi_{M T_{2}}(n)$ - число функций из класса $M_{3}(n) \cap T_{\mathscr{G}_{2}, 1}(n)$. Тогда справедливо равенство

$$
\psi_{M T_{2}}(n)=2^{\sqrt{3 /(4 \pi n)} 3^{n}\left(1+\varepsilon_{4}(n)\right)},
$$

где $\varepsilon_{3}(n) \rightarrow 0$ npu $n \rightarrow \infty$.

Для доказательства этого равенства достаточно провести те же самые рассуждения с взаимной заменой констант 2 на 0 и соответствующей перестановкой порядков.

\section{5. Класс $M_{3}(n) \cap M_{3}^{\prime}(n)$}

Определение 9. Классом $M_{3}^{\prime}(n)$ называется множество функций $\left\{E_{3}^{n} \rightarrow O_{3}^{\prime} E_{3}\right\}$.

Теорема 3. Для числа $\psi_{M M^{\prime}}(n)$ функций из класса $M_{3}(n) \cap M_{3}^{\prime}(n)$ справедливо равенство

$$
\psi_{M M^{\prime}}(n)=2^{\sqrt{2 /(\pi n)} 2^{n}(1+\varepsilon(n))},
$$

где $\varepsilon(n) \rightarrow 0$ nрu $n \rightarrow \infty$. 
Для доказательства теоремы нам потребуется несколько вспомогательных утверждений.

Для сокращения записи обозначим исследуемый класс через F. Введем множество $\hat{A}=\left\{A_{1}, A_{2}, \ldots, A_{2^{n}}\right\}$, состоящее из всех 2-подкубов куба $E_{3}^{n}$.

Лемма 13. Пусть $f \in F_{,}, A-$ произвольный 2-подкуб куба $E_{3}^{n}$. Тогда $f$ на A принимает либо только значение 2, либо только значения 0 и 1 .

Доказательство. Пусть $N$ - индекс $A$. Предположим, что на наборе $\tilde{\alpha} \in A$ функщи $f$ принимает значение 2. Для доказательства леммы достаточно показать, что в этом случае на всем 2-подкубе $A$ принимается значение 2.

Для этого рассмотрим набор $\tilde{\alpha}_{0}=\left(\alpha_{1}, \alpha_{2}, \ldots, \alpha_{n}\right)$, где

$$
\begin{aligned}
& \alpha_{j}=0 \Longleftrightarrow j \notin N, \\
& \alpha_{j}=2 \Longleftrightarrow j \in N .
\end{aligned}
$$

Ясно, что он также принадлежит 2-подкубу $A$.

Из определения порядка $O_{3}^{\prime}$ и того, что функция $f$ сохраняет порядок $O_{3}^{\prime}$, следует, чTO

$$
\tilde{\alpha} \leqslant o_{3}^{\prime} \tilde{\alpha}_{0} \Longrightarrow 2=f(\tilde{\alpha}) \leqslant o_{3}^{\prime} f\left(\tilde{\alpha}_{0}\right) \Longrightarrow f\left(\tilde{\alpha}_{0}\right)=2 \text {. }
$$

С другой стороны, для произвольного набора $\tilde{\beta}$ из $A$

$$
\tilde{\alpha}_{0} \leqslant o_{3} \tilde{\beta} \Longrightarrow 2=f\left(\tilde{\alpha}_{0}\right) \leqslant o_{3} f(\tilde{\beta}) \Longrightarrow f(\tilde{\beta})=2 \text {. }
$$

Таким образом, на всем множестве $\boldsymbol{A}$ будет приниматься значение 2.

Лемма доказана.

Теперь для каждой функщии $f \not \equiv 2$ из $\mathscr{F}$ построим множество $I(f) \subset\{1,2, \ldots, n\}$ следующим образом:

$$
I(f)=\bigcup_{A \in \hat{A}: f(A) \subset\{0,1\}} N(A),
$$

где $N(A)$ - индекс 2-подкуба $A$.

Введем обозначение

$$
\mathscr{F}(I)=\{f \in \mathscr{F} \mid I(f)=I\}
$$

Заметим, что

$$
\mathscr{F}=\left(\biguplus_{I \subset\{1,2, \ldots, n\}} \mathscr{F}(I)\right) \cup\{f \equiv 2\} .
$$

Переформулируем определение монотонных булевых функщий в наших обозначениях.

Определение 10. Классом $M_{2}(n)$ называется множество функщий $\left\{E_{2}^{n} \rightarrow o_{3}^{\circ} E_{2}\right\}$.

Докажем основную лемму.

Лемма 14. Пусть $I \subset\{1,2, \ldots, n\}$. Тогда

$$
|\mathscr{F}(I)| \leqslant\left|M_{2}(|I|)\right|\left|M_{2}(n-|I|)\right| .
$$


Доказательство. Согласно определению 2-подкуба, он однозначно определяется своим индексом. Построим взаимно однозначное соответствие между элементами множества $E_{2}^{n}$ и индексами 2-подкубов и будем считать, что по такому же правилу определяется соответствие между наборами из $E_{2}^{n}$ и 2-подкубами куба $E_{3}^{n}$.

Пусть $\tilde{\alpha}=\left(\alpha_{1}, \alpha_{2}, \ldots, \alpha_{n}\right) \in E_{2}^{n}$. Определим множество $N_{\tilde{\alpha}} \subset\{1,2, \ldots, n\}$, полагая

$$
i \in N_{\tilde{\alpha}} \Longleftrightarrow \alpha_{i}=1, \quad i=1, \ldots, n \text {. }
$$

Пусть $A_{N_{\tilde{\alpha}}}-2$-подкуб с индексом $N_{\tilde{\alpha}}$.

Теперь построим для каждой функии $f$ из $\mathscr{F}(I)$ функщию $h_{f}$, определенную на множестве $E_{2}^{n}$ и задаваемую для всех $\tilde{\alpha} \in E_{2}^{n}$ формулой

$$
h_{f}(\widetilde{\alpha})= \begin{cases}1, & f\left(A_{N_{\tilde{\alpha}}}\right)=\{2\}, \\ 0 & \text { в противном случае }\end{cases}
$$

Покажем, что все такие функции являются монотонными булевыми функциями.

Лемма 15. Пусть $f \in \mathscr{F}(I) u h_{f}$ определяется формулой (12). Тогда $h_{f} \in\left\{E_{2}^{n} \rightarrow o_{3}^{0} E_{2}\right\}$.

Доказательство. По определению $h_{f} \in\left\{E_{2}^{n} \rightarrow E_{2}\right\}$. Покажем, что $h_{f}$ сохраняет $O_{3}^{0}$. Фиксируем произвольные наборы $a, b \in E_{2}^{n}$ такие, что $a \leqslant \sigma_{3}^{0} b$. Очевидно, что если $h_{f}(a)=0$, то $h_{f}(a) \leqslant o_{3}^{0} h_{f}(b)$. Предположим, что $h_{f}(a)=1$. Согласно определению $h_{f}$, это означает, что $f\left(A_{N_{a}}\right)=\{2\}$. Возьмем в 2 -подкубе $A_{N_{a}}$ набор $\tilde{\alpha}=\left(\alpha_{1}, \alpha_{2}, \ldots, \alpha_{n}\right)$ такой, что

$$
\alpha_{i}= \begin{cases}2, & \text { если } i \in N_{a}, \\ 1 & \text { в противном случае. }\end{cases}
$$

Аналогичным образом возьмем в 2 -подкубе $A_{N_{b}}$ набор $\tilde{\beta}=\left(\beta_{1}, \beta_{2}, \ldots, \beta_{n}\right)$ такой, что

$$
\beta_{i}= \begin{cases}2, & \text { если } i \in N_{b} \\ 1 & \text { в противном случае }\end{cases}
$$

Поскольку $a \leqslant o_{3}^{0} b$, справедливо соотношение (11), $N_{a} \subset N_{b}$. Отсюда получаем, что $\tilde{\alpha} \leqslant o_{3} \tilde{\beta}$. Функция $f$ по определению сохраняет порядок $O_{3}$, поэтому $2=f(\tilde{\alpha}) \leqslant o_{3} f(\tilde{\beta})$. Следовательно, $f(\widetilde{\beta})=2$. Согласно лемме 13 это означает, что на всех наборах 2-подкуба $A_{N_{b}}$ функция $f$ принимает только значение 2 . По определению функции $h_{f}$ это означает, что $h_{f}(b)=1$. Таким образом, $h_{f}(a) \leqslant o_{3}^{0} h_{f}(b)$. Лемма доказана.

Введем обозначение

$$
H_{\mathscr{F}_{I}}=\bigcup_{f \in \mathscr{F}(I)}\left\{h_{f}\right\}
$$

Лемма 16. Пусть $I \subset\{1,2, \ldots, n\}$. Тогда

$$
\left|H_{\mathscr{F}_{I}}\right| \leqslant\left|M_{2}(|I|)\right|
$$

Доказательство. В случае, если $I=\{1,2, \ldots, n\}$, утверждение леммы является следствием леммы 15. 
Рассмотрим случай $I \neq\{1,2, \ldots, n\}$. Фиксируем произвольную функцию $f$ из $\mathscr{F}(I)$ и произвольное число $j \in\{1,2, \ldots, n\} \backslash I$. Фиксируем также произвольный набор $\tilde{\alpha}$ куба $E_{2}^{n}$, $j$-я компонента которого равна единище. Рассмотрим соответствующий ему 2-подкуб $A_{N_{\tilde{\alpha}}}$. Согласно определению $N_{\tilde{\alpha}}, j \in N_{\tilde{\alpha}}$. Кроме того, $j \notin I$. Согласно определению $I$ (см. (9)) и лемме 13 на $A_{N_{\widetilde{\alpha}}}$ функщия $f$ будет принимать только значение 2 . По определению $h_{f}$ это дает равенство $h_{f}(\widetilde{\alpha})=1$.

Итак, если в наборе из $E_{2}^{n}$ хотя бы одна компонента с номером не из $I$ равна 1 , то значение функщии $h_{f}$ на этом наборе равно 1 . Пусть $|I|=l$. Не ограничивая общности рассуждений, положим $I=\{1,2, \ldots, l\}$. Тогда получаем, что функцию $h_{f}$ можно представить в виде

$$
h_{f}\left(x_{1}, x_{2}, \ldots, x_{n}\right)=x_{l+1} \vee x_{l+2} \vee \ldots \vee x_{n} \vee \hat{h}_{f}\left(x_{1}, x_{2}, \ldots, x_{l}\right),
$$

где $\hat{h}_{f}$ - тоже монотонная функция, то есть $\hat{h}_{f} \in\left\{E_{2}^{l} \rightarrow o_{3}^{0} E_{2}\right\}$

Число функций вида (13) не превосходит

$$
\left|\left\{E_{2}^{l} \rightarrow o_{3}^{0} E_{2}\right\}\right|=\left|M_{2}(l)\right|=\left|M_{2}(|I|)\right| .
$$

Отсюда следует утверждение леммы.

Теперь рассмотрим произвольную функщию $f$ из класса $\mathscr{F}(I)$. Пусть $D$ - множество наборов куба $E_{3}^{n}$, на которых функция $f$ не принимает значение 2 .

Пусть мощность $|I|$ равна $l$. Не ограничивая общности рассуждений, положим $I=\{1,2, \ldots, l\}$. Пусть $\alpha_{l+1}, \alpha_{l+2}, \ldots, \alpha_{n}$ - элементы множества $\{0,1\}$. Введем множество наборов

$$
B\left(\alpha_{l+1}, \alpha_{l+2}, \ldots, \alpha_{n}\right)=\left\{\left(a_{1}, a_{2}, \ldots, a_{n}\right) \in D \mid a_{l+1}=\alpha_{l+1}, a_{l+2}=\alpha_{l+2}, \ldots, a_{n}=\alpha_{n}\right\} .
$$

Лемма 17. Пусть $f-$ функция из $\mathscr{F}(I), a \hat{\alpha}=\left(\alpha_{l+1}, \alpha_{l+2}, \ldots, \alpha_{n}\right)-$ набор элементов множества $\{0,1\}$. Пусть $а$ и $b-$ наборы из $E_{3}^{n}$ такие, что $b \in B(\hat{\alpha})$, $a=\left(a_{1}, a_{2}, \ldots, a_{l}, \hat{\alpha}\right) u a \leqslant o_{3} b$. Toгдa $a \in B(\widehat{\alpha})$.

Доказательство. Достаточно доказать, что $a \in D$. Действительно, $b \in B(\hat{\alpha})$, следовательно, $f(b) \in\{0,1\}$. Функщия $f$ сохраняет порядок $O_{3}$, а $a \leqslant o_{3} b$, поэтому $f(a) \leqslant o_{3} f(b)$. Отсюда получаем, что $f(a) \in\{0,1\}$, следовательно, $a \in D$.

Лемма 18. Пусть $f-$ функция из $\mathscr{F}(I), a \hat{\alpha}=\left(\alpha_{l+1}, \alpha_{l+2}, \ldots, \alpha_{n}\right)-$ набор элементов множества $\{0,1\}$. Пусть $a=\left(a_{1}, a_{2}, \ldots, a_{n}\right), b=\left(b_{1}, b_{2}, \ldots, b_{n}\right)-$ наборы из $B(\hat{\alpha})$, причем

$$
a_{i} \neq b_{i} \Longleftrightarrow b_{i}=2, \quad i=1, \ldots, n
$$

Тогда $f(a)=f(b)$.

Доказательство. Заметим, что $a \leqslant o_{3} b$ и $a \leqslant o_{3}^{\prime} b$. Отсюда следует, что справедливы соотношения $f(a) \leqslant o_{3} f(b)$ и $f(a) \leqslant o_{3}^{\prime} f(b)$. Набор $b$ лежит в $B\left(\alpha_{l+1}, \alpha_{l+2}, \ldots, \alpha_{n}\right)$, поэтому $f(b) \in\{0,1\}$. Заметим, что 0 - минимальный элемент порядка $O_{3}$, а 1 - минимальный элемент порядка $O_{3}^{\prime}$. Из этого следует, что $f(b)$ является минимальным элементом по одному из порядков. Отсюда получаем, что $f(a)=f(b)$.

Лемма 19. Пусть $f$ - функция из $\mathscr{F}(I), a \alpha_{l+1}, \alpha_{l+2}, \ldots, \alpha_{n}-$ элементы множества $\{0,1\}$. Тогда на множестве $B\left(\alpha_{l+1}, \alpha_{l+2}, \ldots, \alpha_{n}\right)$ функция $f$ либо тождественно равна 0 , либо тождественно равна 1. 
Доказательство. Для краткости обозначим $\widehat{\alpha}$ последовательность $\alpha_{l+1}, \alpha_{l+2}, \ldots, \alpha_{n}$. Так как множество $B(\widehat{\alpha})$ является подмножеством $D$, функция $f$ на нем принимает только значения 0 и 1 . Поэтому для доказательства леммы достаточно показать, что функция $f$ постоянна на $B(\hat{\alpha})$.

Для зтого индукцией по $m$ докажем, что для любого $\left(\beta_{1}, \beta_{2}, \ldots, \beta_{l}, \hat{\alpha}\right) \in B(\widehat{\alpha})$ справедливо равенство

$$
f\left(\beta_{1}, \beta_{2}, \ldots, \beta_{l}, \hat{\alpha}\right)=f\left(0, \ldots, 0, \beta_{m+1}, \beta_{m+2}, \ldots, \beta_{l}, \widehat{\alpha}\right),
$$

где в правой части равны нулю первые $m$ аргументов функции $f$.

Очевидно, при $m=0$ равенство (14) верно.

Предположим, что при всех $m<t \leqslant l$ равенство (14) выполнено. Докажем равенство для $m=t$.

Фиксируем произвольный набор $\widetilde{\beta}=\left(\beta_{1}, \beta_{2}, \ldots, \beta_{l}, \widehat{\alpha}\right)$ из $B(\widehat{\alpha})$. Рассмотрим следующие случаи.

Пусть $\beta_{t}=0$. Тогда по предположению индукции

$$
\begin{aligned}
f\left(\beta_{1}, \ldots, \beta_{t-1}, \beta_{t}, \beta_{t+1}, \ldots, \beta_{l}, \widehat{\alpha}\right) & =f(\underbrace{0, \ldots, 0}_{t-1}, \beta_{t}, \beta_{t+1}, \ldots, \beta_{l}, \widehat{\alpha}) \\
& =f(\underbrace{0, \ldots, 0}_{t-1}, 0, \beta_{t+1}, \ldots, \beta_{l}, \widehat{\alpha}) \\
& =f(\underbrace{0, \ldots, 0}_{t}, \beta_{t+1}, \beta_{t+2}, \ldots, \beta_{l}, \widehat{\alpha}),
\end{aligned}
$$

что и требовалось доказать.

Пусть $\beta_{t}=2$. Тогда рассмотрим набор $\tilde{\beta}_{0}=\left(\beta_{1}, \ldots, \beta_{t-1}, 0, \beta_{t+1}, \ldots, \beta_{l}, \hat{\alpha}\right)$. Для пары наборов $\widetilde{\beta}_{0}$ и $\widetilde{\beta}$ верно утверждение леммы 17, поэтому $\widetilde{\beta}_{0} \in B(\widehat{\alpha})$. В этом случае для $\widetilde{\beta}_{0}$ и $\widetilde{\beta}$ верно также утверждение леммы 18 , поэтому $f(\widetilde{\beta})=f\left(\widetilde{\beta}_{0}\right)$. Кроме того, по предположению индукции

$$
f\left(\tilde{\beta}_{0}\right)=f(\underbrace{0, \ldots, 0}_{t}, \beta_{t+1}, \beta_{t+2}, \ldots, \beta_{l}, \widehat{\alpha}),
$$

и в этом случае мы также доказали верность индуктивного перехода.

Пусть теперь $\beta_{t}=1$. Рассмотрим набор $\widetilde{\beta}^{\prime}=\left(\beta_{1}^{\prime}, \beta_{2}^{\prime}, \ldots, \beta_{l}^{\prime}, \widehat{\alpha}\right)$, где $\beta_{i}^{\prime}, i=1, \ldots, l$, определяются по правилу

$$
\beta_{i}^{\prime}= \begin{cases}0, & \text { если } \beta_{i}=2, \\ \beta_{i} & \text { в противном случае. }\end{cases}
$$

Заметим, что для наборов $\widetilde{\beta}$ и $\widetilde{\beta}^{\prime}$ верно утверждение леммы 17 , поэтому $\widetilde{\beta}^{\prime} \in B(\widehat{\alpha})$. В этом случае для $\widetilde{\beta}$ и $\widetilde{\beta}^{\prime}$ верно также утверждение леммы 18 , поэтому

$$
f(\tilde{\beta})=f\left(\tilde{\beta}^{\prime}\right) .
$$

Константа $t$ лежит в множестве $I$, что означает, что существует такой 2-подкуб $A$, индекс $N \subset I$ которого содержит $t$, причем $f(A) \subset\{0,1\}$. Таким образом, построив набор $\widetilde{\beta}^{A}=\left(\beta_{1}^{A}, \beta_{2}^{A}, \ldots, \beta_{l}^{A}, \widehat{\alpha}\right)$ из этого 2-подкуба такой, что для $i=1, \ldots, l$

$$
\beta_{i}^{A}= \begin{cases}2, & \text { если } i \in N ; \\ \beta_{i}^{\prime} & \text { в противном случае }\end{cases}
$$


мы получим, что $\widetilde{\beta}^{A} \in B(\widehat{\alpha})$. Заметим, что для наборов $\tilde{\beta}^{\prime}$ и $\tilde{\beta}^{A}$ верно утверждение леммы 18 , поэтому

$$
f\left(\tilde{\beta}^{\prime}\right)=f\left(\tilde{\beta}^{A}\right)
$$

Построим набор $\tilde{\beta}^{\prime \prime}=\left(\beta_{1}^{\prime \prime}, \beta_{2}^{\prime \prime}, \ldots, \beta_{l}^{\prime \prime}, \widehat{\alpha}\right)$, где $\beta_{i}^{\prime \prime}, i=1, \ldots, l$, определяются по правилу $\beta_{t}^{\prime \prime}=0, \beta_{i}^{\prime \prime}=\beta_{i}^{\prime}$, если $i \neq t$.

Так как $t \in N$ и $\widetilde{\beta}^{\prime \prime} \in B(\widehat{\alpha})$ (достаточно применить лемму 17), для наборов $\widetilde{\beta}^{A}$ и $\tilde{\beta}^{\prime \prime}$ верно утверждение леммы 18 , поэтому

$$
f\left(\tilde{\beta}^{A}\right)=f\left(\tilde{\beta}^{\prime \prime}\right)
$$

Наконец, построим набор $\tilde{\beta}^{\prime \prime \prime}=\left(\beta_{1}^{\prime \prime \prime}, \beta_{2}^{\prime \prime \prime}, \ldots, \beta_{l}^{\prime \prime \prime}, \widehat{\alpha}\right)$, где $\beta_{i}^{\prime \prime \prime}, i=1, \ldots, l$, определяются по правилу $\beta_{t}^{\prime \prime \prime}=0, \beta_{i}^{\prime \prime \prime}=\beta_{i}$, если $i \neq t$.

Заметим, что $\widetilde{\beta}^{\prime \prime \prime} \leqslant o_{3} \widetilde{\beta}$, поэтому по лемме $17 \widetilde{\beta}^{\prime \prime \prime} \in B(\widehat{\alpha})$. В силу (15) получаем, что для наборов $\tilde{\beta}^{\prime \prime}$ и $\tilde{\beta}^{\prime \prime \prime}$ верно утверждение леммы 18 , поэтому

$$
f\left(\tilde{\beta}^{\prime \prime}\right)=f\left(\tilde{\beta}^{\prime \prime \prime}\right) \text {. }
$$

По построению

$$
f\left(\tilde{\beta}^{\prime \prime \prime}\right)=f\left(\beta_{1}, \ldots, \beta_{t-1}, 0, \beta_{t+1}, \ldots, \beta_{l}, \widehat{\alpha}\right) .
$$

В то же время по предположению индукции

$$
\begin{aligned}
f\left(\tilde{\beta}^{\prime \prime \prime}\right) & =f\left(\beta_{1}, \ldots, \beta_{t-1}, 0, \beta_{t+1}, \ldots, \beta_{l}, \widehat{\alpha}\right) \\
& =f(\underbrace{0, \ldots, 0}_{t-1}, 0, \beta_{t+1}, \beta_{t+2}, \ldots, \beta_{l}, \widehat{\alpha}) \\
& =f(\underbrace{0, \ldots, 0}_{t}, \beta_{t+1}, \beta_{t+2}, \ldots, \beta_{l}, \widehat{\alpha}) .
\end{aligned}
$$

Объединяя равенства (16), (17), (18), (19) и (20), получим, что

$$
f(\tilde{\beta})=f\left(\beta_{1}, \ldots, \beta_{t-1}, \beta_{t}, \beta_{t+1}, \ldots, \beta_{l}, \hat{\alpha}\right)=f(\underbrace{0, \ldots, C}_{t}, \beta_{t+1}, \beta_{t+2}, \ldots, \beta_{l}, \hat{\alpha}) .
$$

Таким образом, и в последнем случае индуктивный переход имеет место.

Этим завершается доказательство индуктивного перехода. Вспомогательное утверждение доказано. Взяв $m=l$, получим, что для всех $\left(\beta_{1}, \beta_{2}, \ldots, \beta_{l}, \hat{\alpha}\right) \in B(\widehat{\alpha})$

$$
f\left(\beta_{1}, \beta_{2}, \ldots, \beta_{l}, \widehat{\alpha}\right)=f(\underbrace{0, \ldots, 0}_{l}, \widehat{\alpha}),
$$

что и дает утверждение леммы.

Лемма доказана.

Пусть $f \in \mathscr{F}(I)$. Определим функцию $s_{f}$, действующую на множестве $E_{2}^{n-l}$, по правилу

$$
s_{f}\left(\alpha_{1}, \alpha_{2}, \ldots, \alpha_{n-l}\right)=f(\underbrace{0, \ldots, 0}_{l}, \alpha_{1}, \alpha_{2}, \ldots, \alpha_{n-l}) .
$$

Введем функциональное множество

$$
S_{\mathscr{F}_{g}}=\left\{E_{2}^{n-l} \rightarrow{ }_{O_{3}^{0}} E_{2}\right\}
$$


Лемма 20. Пусть $f \in \mathscr{F}(I), s_{f}$ определяется по правилу (21). Тогда $s_{f} \in S_{\mathscr{F}_{s}}$.

Доказательство. Сначала покажем, что $s_{f}$ действует в $E_{2}$. Фиксируем произвольный набор $\widetilde{\beta} \in E_{2}^{n-l}$.

По определению $I$ и $\mathscr{F}(I)$, функщия $f$ тождественно не равна 2 , следовательно, существует набор $\tilde{\alpha} \in E_{3}^{n}$ такой, что $f(\widetilde{\alpha}) \in\{0,1\}$. Заметим, что $\widetilde{\alpha}_{0} \leqslant o_{3} \tilde{\alpha}$. Функщия $f$ принадлежит классу $M_{3}(n)$, поэтому $f\left(\widetilde{\alpha}_{0}\right) \leqslant o_{3} f(\widetilde{\alpha})$. Отсюда получаем, что $f\left(\widetilde{\alpha}_{0}\right) \in\{0,1\}$. Набор $\widetilde{\alpha}_{0}$ находится в одном 2-подкубе с набором $(0, \ldots, 0, \widetilde{\beta})$ размерности $n$ и по лемме $13 f(0, \ldots, 0, \widetilde{\beta})$ также лежит во множестве $\{0,1\}$. Следовательно, $s_{f}(\widetilde{\beta}) \in\{0,1\}=E_{2}$.

Покажем, что построенная функщия $s_{f}$ сохраняет порядок $O_{3}^{0}$. Пусть

$$
\left(a_{1}, a_{2}, \ldots, a_{n-l}\right), \quad\left(b_{1}, b_{2}, \ldots, b_{n-l}\right) \in E_{2}^{n-l},
$$

причем

$$
\left(a_{1}, a_{2}, \ldots, a_{n-l}\right) \leqslant o_{3}^{0}\left(b_{1}, b_{2}, \ldots, b_{n-l}\right)
$$

В этом случае

$$
\begin{aligned}
& \left(a_{1}, a_{2}, \ldots, a_{n-l}\right) \leqslant o_{3}^{0}\left(b_{1}, b_{2}, \ldots, b_{n-l}\right) \\
& (\underbrace{0, \ldots, 0}_{l}, a_{1}, a_{2}, \ldots, a_{n-l}) \leqslant o_{3}(\underbrace{0, \ldots, 0}_{l}, b_{1}, b_{2}, \ldots, b_{n-l}) \\
& f(\underbrace{0, \ldots, 0}_{l}, a_{1}, a_{2}, \ldots, a_{n-l}) \leqslant o_{3} f(\underbrace{0, \ldots, 0}_{l}, b_{1}, b_{2}, \ldots, b_{n-l}) \\
& s_{f}\left(a_{1}, a_{2}, \ldots, a_{n-l}\right) \leqslant o_{3} s_{f}\left(b_{1}, b_{2}, \ldots, b_{n-l}\right) \\
& s_{f}\left(a_{1}, a_{2}, \ldots, a_{n-l}\right) \leqslant o_{3}^{0} s_{f}\left(b_{1}, b_{2}, \ldots, b_{n-l}\right) .
\end{aligned}
$$

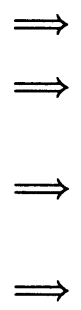

Лемма доказана.

Таким образом, определив отображение $\mathscr{P}$ на множестве $\mathscr{F}(I)$ по правилу $\mathscr{P}(f)=h_{f} \times s_{f}$, получим, что $\mathscr{P}$ действует из $\mathscr{F}(I)$ в $H_{\mathscr{F}_{g}} \times S_{\mathscr{F}_{I}}$.

Лемма 21. Существует инъективное отображение $\mathscr{P}$ из $\mathscr{F}_{(1)}(I) H_{\mathscr{F}_{9}} \times S_{\mathscr{F}_{I}}$.

Доказательство. Для произвольной функщии $f$ из $\mathscr{F}(I)$ положим

$$
\mathscr{P}(f)=\left(h_{f}, s_{f}\right),
$$

где $h_{f}$ определяется по правилу (12), а $s_{f}$ по правилу (21). Согласно определению $H_{\xi_{g}}$ и лемме 20 , это отображение действует в $H_{\mathscr{F}_{g}} \times S_{\mathscr{F}_{I}}$. Покажем его инъективность.

Действительно, возьмем две любые различные функции $f^{\prime}$ и $f^{\prime \prime}$ из F्F( $(I)$. Пусть набор $\tilde{\alpha}$ их различает. Возможны следующие варианты.

Среди значений $f^{\prime}(\widetilde{\alpha}), f^{\prime \prime}(\widetilde{\alpha})$ есть двойка. Пусть $A_{\tilde{\alpha}}-2$-подкуб, соответствующий набору $\widetilde{\alpha}$. По лемме 13 , одна из функций $f^{\prime}, f^{\prime \prime}$ будет принимать на нем только значение 2 , а другая только значения 0 и 1 . Определим набор $a=\left(a_{1}, a_{2}, \ldots, a_{n}\right)$ из множества $E_{2}^{n}$ по правилу

$$
a_{i}= \begin{cases}1, & \text { если } j \in N\left(A_{\tilde{\alpha}}\right), \\ 0 & \text { в противном случае. }\end{cases}
$$

В этом случае $A_{N_{a}}=A_{\tilde{\alpha}}$. Согласно определению, одна из функций $h_{f^{\prime}}, h_{f^{\prime \prime}}$ на $a$ принимает значение 1 , а другая значение 0 , поэтому $h_{f^{\prime}} \neq h_{f^{\prime \prime}}$. В этом случае $\mathscr{P}\left(f^{\prime}\right) \neq \mathscr{P}\left(f^{\prime \prime}\right)$. 
Среди значений $f^{\prime}(\widetilde{\alpha}), f^{\prime \prime}(\widetilde{\alpha})$ нет двойки, следовательно, есть 0 и 1 . Пусть

$$
\tilde{\alpha}=\left(\alpha_{1}, \alpha_{2}, \ldots, \alpha_{n}\right), \quad \tilde{\alpha}_{0}=(\underbrace{0, \ldots, 0}_{l}, \alpha_{l+1}, \ldots, \alpha_{n}) .
$$

В этом случае $\tilde{\alpha}, \tilde{\alpha}_{0} \in B\left(\alpha_{l+1}, \alpha_{l+2}, \ldots, \alpha_{n}\right)$. Согласно лемме 19

$$
f^{\prime}\left(\tilde{\alpha}_{0}\right)=f^{\prime}(\tilde{\alpha}), \quad f^{\prime \prime}\left(\tilde{\alpha}_{0}\right)=f^{\prime \prime}(\tilde{\alpha}) .
$$

По определению функщии $s_{f}$

$$
s_{f^{\prime}}\left(\alpha_{l+1}, \alpha_{l+2}, \ldots, \alpha_{n}\right)=f^{\prime}\left(\widetilde{\alpha}_{0}\right), \quad s_{f^{\prime \prime}}\left(\alpha_{l+1}, \alpha_{l+2}, \ldots, \alpha_{n}\right)=f^{\prime \prime}\left(\widetilde{\alpha}_{0}\right),
$$

откуда следует, что $s_{f^{\prime}} \neq s_{f^{\prime \prime}}$. Таким образом, и в этом случае $\mathscr{P}\left(f^{\prime}\right) \neq \mathscr{P}\left(f^{\prime \prime}\right)$. Лемма доказана.

Из лемм 21 и 16 следует, что для $\mathscr{F}(I)$ справедлива оценка

$|F(I)| \leqslant\left|H_{\mathscr{F}_{g}}\right|\left|S_{\mathscr{F}_{g}}\right| \leqslant\left\{M_{2}(n-l)=S_{\mathscr{F}_{I}}\right\} \leqslant\left|M_{2}(l)\right|\left|M_{2}(n-l)\right|=\left|M_{2}(|I|)\right|\left|M_{2}(n-|I|)\right|$.

Основная лемма доказана.

Лемма 22. Справедлива оченка

$$
|F| \leqslant 2^{\sqrt{2 /(\pi n)} 2^{n}\left(1+\varepsilon_{1}(n)\right)},
$$

где $\varepsilon_{1}(n) \rightarrow 0$ npu $n \rightarrow \infty$.

Доказательство. Согласно основной лемме и равенству (10) справедливы неравенства

$$
\begin{aligned}
|\mathscr{F}| & \leqslant 1+\sum_{k=0}^{n} \sum_{|I|=k}|\mathscr{F}(I)| \leqslant 1+\sum_{k=0}^{n}\left(\begin{array}{l}
n \\
k
\end{array}\right)\left|M_{2}(k)\right|\left|M_{2}(n-k)\right| \\
& \leqslant 2\left(\begin{array}{c}
n \\
\lfloor n / 2\rfloor
\end{array}\right) \sum_{k=0}^{\lfloor n / 2\rfloor}\left|M_{2}(k)\right|\left\|M_{2}(n-k)\left|\leqslant n\left(\begin{array}{c}
n \\
\lfloor n / 2\rfloor
\end{array}\right)\right| M_{2}(\lfloor n / 2\rfloor)\right\| M_{2}(n) \mid .
\end{aligned}
$$

Согласно [3] для $\left|M_{2}(n)\right|$ справедлива оценка

$$
\left|M_{2}(n)\right|=2^{\sqrt{2 /(\pi n)} 2^{n}\left(1+\varepsilon_{2}(n)\right)},
$$

где $\varepsilon_{2}(n) \rightarrow 0$ при $n \rightarrow \infty$. Отсюда получаем, что

$$
\begin{aligned}
|F| F \mid & \leqslant n\left(\begin{array}{c}
n \\
\lfloor n / 2\rfloor
\end{array}\right)\left|M_{2}(\lfloor n / 2\rfloor)\right|\left|M_{2}(n)\right| \\
& \leqslant 2^{n+\log _{2} n+\sqrt{2 /(\pi n / 2)} 2^{n / 2}\left(1+\varepsilon_{2}(n)\right)+\sqrt{2 /(\pi n) 2^{n}}\left(1+\varepsilon_{2}(n)\right)}=2^{\sqrt{2 /(\pi n)} 2^{n}\left(1+\varepsilon_{1}(n)\right)},
\end{aligned}
$$

где $\varepsilon_{1}(n) \rightarrow 0$ при $n \rightarrow \infty$. Лемма доказана.

Лемма 23. Существует инъективное отображение 2 из $S_{\mathscr{F}_{\varnothing}}$ в $\mathscr{F}(\varnothing)$. 
Доказательство. Напомним, что по определению

$$
S_{\Phi_{\varnothing}}=\left\{E_{2}^{n} \rightarrow_{O_{3}^{0}} E_{2}\right\} .
$$

Построим для произвольной функции $f \in S_{\oiint_{\varnothing}}$ функцию $f^{\prime}=2[f]$, действующую на $E_{3}^{n}$, по следующему правилу: для любого $\widetilde{\alpha}=\left(\alpha_{1}, \alpha_{2}, \ldots, \alpha_{n}\right) \in E_{3}^{n}$

$$
f^{\prime}(\widetilde{\alpha})= \begin{cases}2, & \text { если существует } i \text { такое, что } \alpha_{i}=2, \\ f(\widetilde{\alpha}) & \text { в противном случае. }\end{cases}
$$

Очевидно, что все построенные таким образом функции различны. Покажем, что все они принадлежат классу $\mathscr{F}(\varnothing)$. По определению $\mathscr{F}(\varnothing)$ в этот класс входят все функщии класса $\mathscr{F}$, отличные от двойки только на 2-подкубе с параметром $N=\varnothing$.

Так как построенные функщии отличны от 2 только на 2-подкубе с параметром $N=\varnothing$ (обозначим его $A_{0}$ ), достаточно показать, что все они сохраняют порядки $O_{3}$ и $O_{3}^{\prime}$. Фиксируем два произвольных набора $a \leqslant o_{3} b$ и функцию $f \in S_{\Im_{\varnothing}}$. Возможны следующие варианты.

(1) $a \in A_{0}, b \notin A_{0}$. Тогда $f^{\prime}(a)=f(a) \in\{0,1\}$ и

$$
f^{\prime}(b)=2 \Longrightarrow f^{\prime}(a) \leqslant o_{3} f^{\prime}(b) .
$$

(2) $a \notin A_{0}, b \notin A_{0}$. Тогда

$$
f^{\prime}(a)=f^{\prime}(b)=2 \Longrightarrow f^{\prime}(a) \leqslant o_{3} f^{\prime}(b) .
$$

(3) $a \in A_{0}, b \in A_{0}$. Тогда $f^{\prime}(a)=f(a), f^{\prime}(b)=f(b)$. Так как $f$ сохраняет порядок $O_{3}^{0}$, a $a \leqslant o_{3} b \Rightarrow a \leqslant{o_{3}^{0}}_{3} b$, справедливы соотношения

$$
f(a) \leqslant o_{3}^{0} f(b) \Longrightarrow f(a) \leqslant o_{3} f(b) \Longrightarrow f^{\prime}(a) \leqslant o_{3} f^{\prime}(b) .
$$

(4) $a \notin A_{0}, b \in A_{0}$. Это означает, что некоторая (скажем, $i$-я) компонента набора $a$ равна 2. Но $a \leqslant o_{3} b$, поэтому $i$-я компонента набора $b$ также должна быть равна 2, что противоречит тому, что $b \in A_{0}$. Поэтому такой случай невозможен.

Теперь фиксируем произвольные наборы $a \leqslant o_{3}^{\prime} b$. Рассмотрим те же варианты.

(1') $a \in A_{0}, b \notin A_{0}$. Тогда $f^{\prime}(a)=f(a) \in\{0,1\}$ и

$$
f^{\prime}(b)=2 \Longrightarrow f^{\prime}(a) \leqslant o_{3}^{\prime} f^{\prime}(b) .
$$

(2') $a \notin A_{0}, b \notin A_{0}$. Тогда

$$
f^{\prime}(a)=f^{\prime}(b)=2 \Longrightarrow f^{\prime}(a) \leqslant o_{3}^{\prime} f^{\prime}(b) .
$$

$\left(3^{\prime}\right) a \in A_{0}, b \in A_{0}$. Тогда $f^{\prime}(a)=f(a), f^{\prime}(b)=f(b)$. Так как $f$ сохраняет порядок $O_{3}^{0}$, a $\left.a \leqslant o_{3}^{\prime} b \Rightarrow b \leqslant_{O_{3}^{0}} a\right)$, справедливы соотношения

$$
f(b) \leqslant o_{3}^{0} f(a) \Longrightarrow f(a) \leqslant o_{3}^{\prime} f(b) \Longrightarrow f^{\prime}(a) \leqslant o_{3}^{\prime} f^{\prime}(b) .
$$


(4') $a \notin A_{0}, b \in A_{0}$. Это означает, что некоторая (скажем, $i$-я) компонента набора $a$ равна 2 . Но $a \leqslant o_{3}^{\prime} b$, поэтому $i$-я компонента набора $b$ также должна быть равна 2 , что противоречит тому, что $b \in A_{0}$. Поэтому такой случай невозможен.

Лемма доказана.

Лемма 24. Справедлива оченка

$$
|\mathscr{F}| \geqslant 2^{\sqrt{2 /(\pi n) \cdot 2^{n}}\left(1+\varepsilon_{2}(n)\right)},
$$

где $\varepsilon_{2}(n) \rightarrow 0$ npu $n \rightarrow \infty$.

Доказательство. Согласно лемме 23, существует инъективное отображение 2, действующее из $S_{\mathscr{F}_{\varnothing}}$ в $\mathscr{F}(\varnothing)$. Поэтому согласно (22)

$$
|\mathscr{F}| \geqslant|\mathscr{F}(\varnothing)| \geqslant\left|S_{\mathscr{F}_{\varnothing}}\right|=\left|M_{2}(n)\right|=2^{\sqrt{2 /(\pi n) 2^{n}}\left(1+\varepsilon_{2}(n)\right)},
$$

где $\varepsilon_{2}(n) \rightarrow 0$ при $n \rightarrow \infty$. Отсюда получаем, что

$$
|F| \geqslant 2^{\sqrt{2 /(\pi n)} 2^{n}\left(1+\varepsilon_{2}(n)\right)},
$$

где $\varepsilon_{2}(n) \rightarrow 0$ при $n \rightarrow \infty$.

Лемма доказана.

Из лемм 24 и 22 получаем утверждение теоремы. Теорема доказана.

Аналогичный результат верен для всех классов, являюшихся пересечением двух предполных классов монотонных функщий.

\section{Список литературы}

1. Яблонский С. В., Функциональные построения в $k$-значной логике. Tруды Матем. ин-та им. В. А. Стеклова АН СССР (1958) 51, 5-142.

2. Алексеев В. Б., О числе монотонных $k$-значных функций. Проблемы кибернетики (1974) 28 , 5-24.

3. Клейтмен Д., О проблеме Дедекинда: число монотонных булевых функций. Киберн. сб. (1970) 7, 43-52.

Статья поступила 28.05.2005. 